\title{
EXISTENCE OF INFINITELY MANY SOLUTIONS FOR SUBLINEAR ELLIPTIC PROBLEMS
}

\author{
X. ZHONG \\ Department of Mathematical Sciences, Tsinghua University, Beijing 100084, China \\ e-mail: zhongxuexiu1989@163.com \\ and W. ZOU* \\ Department of Mathematical Sciences, Tsinghua University, Beijing 100084, China \\ e-mail:wzou@math.tsinghua.edu.cn
}

(Received 3 May 2011; accepted 16 October 2011; first published online 30 March 2012)

Abstract. We study the following nonlinear Dirichlet boundary value problem:

$$
-\Delta u=g(x, u), \quad u \in H_{0}^{1}(\Omega),
$$

where $\Omega$ is a bounded domain in $\mathbb{R}^{N}(N \geq 2)$ with a smooth boundary $\partial \Omega$ and $g \in$ $\mathbf{C}(\Omega \times \mathbb{R})$ is a function satisfying $\lim _{|t| \rightarrow 0} \frac{g(x, t)}{t}=\infty$ for all $x \in \Omega$. Under appropriate assumptions, we prove the existence of infinitely many solutions when $g(x, t)$ is not odd in $t$.

2000 Mathematics Subject Classification. 35J20, 35J25, 35J60.

1. Introduction. We study the following nonlinear Dirichlet boundary value problem:

$$
-\Delta u=g(x, u), \quad u \in H_{0}^{1}(\Omega),
$$

where $\Omega$ is a bounded domain in $\mathbb{R}^{N}(N \geq 2)$ with a smooth boundary $\partial \Omega$ and $g \in \mathbf{C}(\Omega \times \mathbb{R})$ is a function satisfying $\lim _{|t| \rightarrow 0} \frac{g(x, t)}{t} \rightarrow \infty$ for all $x \in \Omega$. This kind of problem arises in many physical and mechanical problems, and was investigated by several authors (see $[\mathbf{1}, \mathbf{4}, \mathbf{7}, \mathbf{8}, \mathbf{9}, \mathbf{1 1}, \mathbf{1 2}])$. In [1], Brezis and Ambrosetti considered the problem

$$
-\Delta u=\mu|u|^{q-1} u+v|u|^{p-1} u, \quad u \in H_{0}^{1}(\Omega),
$$

where $0<q<1<p<2^{*}-1$ but $0<\mu \ll v=1$. In [4], Bartsh and Willem established the existence of infinitely many solutions of Problem (1.2) for every $\mu>0$ and $v \in \mathbb{R}$. In [7], the conditions of $v=1,0<q<1, p=2^{*}-1$ have been considered by Garcia and Peral. In these papers, oddness of $g(x, t)$ in $t$ plays a crucial role to ensure the existence of infinitely many solutions and the global property of $g(x, t)$ was used in an essential way to derive multiplicity results of solutions with negative energy.

*Supported by NSFC (11025106, 10871109). Corresponding author. 
In 2001, Wang [11] considered the problem

$$
-\Delta u=\lambda|u|^{q-1} u+f(x, u), \quad u \in H_{0}^{1}(\Omega),
$$

where $\lambda>0,0<q<1, f(x, t) \in \mathbf{C}(\Omega \times \mathbb{R}, \mathbb{R})$ is odd in $t$ for $|t|$ small and

$$
f(x, t)=o\left(|t|^{q}\right), \text { as }|t| \rightarrow 0, \quad \text { uniformly in } x \in \Omega .
$$

The oddness of $f(x, t)$ in $t$ is still indispensable despite there is no condition imposed on $f(x, t)$ for $t$ large. In [8], Hirano made a breakthrough. He considered the nonlinear function $g(x, t)$ which is not necessarily odd in $t$. His conclusions based on the following condition:

(A) there exist positive numbers $p, q$ and $a$ such that $0<q<1<p<2^{*}-1, N(1-$ $q) /(1+q)<p-1$ and that

$$
\limsup _{|t| \rightarrow 0}\left|\frac{g(x, t)-a|t|^{q-1} t}{|t|^{p}}\right|<\infty \quad \text { uniformly in } x \in \Omega .
$$

Under this condition, he got infinitely many solutions. In his arguments, the strict inequality $N(1-q) /(1+q)<p-1$ is essential. At the end of [8], the author proposed an open problem: whether the condition $N(1-q) /(1+q)<p-1$ can be removed or not? In the present paper, we are going to give a partial answer to this question under suitable conditions. Precisely, we consider the case: $N(1-q) /(1+q)=p-1$.

To introduce our assumptions, we put, for simplicity, $H=H_{0}^{1}(\Omega)$, and denote by $\lambda_{1}<\lambda_{2} \leq \lambda_{3} \leq \cdots$ the sequence of eigenvalues of the problem: $-\Delta v=\lambda v, v \in H$; $|\cdot|_{q}$ stands for the norm of $L^{q}(\Omega)$ for $q>1$; $\| \cdot||$ stands for the norm of $H$ defined by $\|z\|^{2}=|\nabla z|_{2}^{2}$ for $z \in H$. It is known (cf. [6]) that there exists $T>0$ such that $\lim _{k \rightarrow \infty} \lambda_{k} / k^{2 / N}=T$. We now impose the following conditions on $g \in \mathbf{C}(\Omega \times \mathbb{R})$ :

(B) there exist positive numbers $p, q$, $a$ and $\delta$ such that $0<q<1<p<2^{*}-1$, $N(1-q) /(1+q)=p-1$, and

$$
\limsup _{|t| \rightarrow 0}\left|\frac{g(x, t)-a|t|^{q-1} t}{|t|^{p}}\right|<\delta<a^{\frac{2-p}{2-q}} \min \left\{b_{0}, b_{1}\right\}
$$

uniformly in $x \in \Omega$, where

$$
\begin{gathered}
b_{0}:=\frac{(1+p)(1+q)}{4 N(2 p+3 q+7)} T^{\frac{N}{2}} M^{-(1+p)}|\Omega|^{-\frac{p-1}{2}} \\
b_{1}:=M^{-\frac{2(p-q)}{1-q}}|\Omega|^{-\frac{(p-1)(p-q)}{(p+1)(1-q)}}\left(\left(\frac{1-q}{p-1}\right)^{\frac{p-1}{p-q}}+\left(\frac{1-q}{p-1}\right)^{\frac{q-1}{p-q}}\right)^{-\frac{p-q}{1-q}},
\end{gathered}
$$

and $M>0$ is the best constant satisfying $|z|_{p+1} \leq M|| z||$ for all $z \in H$, and $|\Omega|$ denotes the Lebesgue measure of $\Omega$.

The main result is the following:

THEOREM 1.1. Suppose that (B) holds. Then, problem (1.1) possesses a sequence of weak solutions $\left(u_{n}\right) \in H$ such that $\left\|u_{n}\right\|_{L^{\infty}(\Omega)} \rightarrow 0$ as $n \rightarrow \infty$. Moreover, $J\left(u_{n}\right)<0$ and 
$J\left(u_{n}\right) \rightarrow 0$ as $n \rightarrow \infty$, where,

$$
J(v)=\int_{\Omega} \frac{1}{2}|\nabla v|^{2} d x-\int_{\Omega} G(x, v) d x, \quad v \in H,
$$

with $G(x, t)=\int_{0}^{t} g(x, s) d s$.

REMARK 1.1. If (A) holds, we put $p_{1}=N(1-q) /(1+q)+1$, then $p_{1}<p$. Hence, $\lim \sup _{|t| \rightarrow 0}\left|\frac{g(x, t)-a|t|^{q-1} t}{|t|^{p_{1}}}\right|=0$, which implies (B). Then, the results of [8] are contained in our conclusions.

2. Proof of Theorem 1.1. Let $g(x, t)=a|t|^{q-1} t+f(x, t), \lambda=a^{\frac{1}{2-q}}, u=\lambda v$, then the problem (1.1) is equivalent to $-\Delta v=|v|^{q-1} v+a^{-\frac{2}{2-q}} f\left(x, a^{\frac{1}{2-q}} v\right), v \in H$. Hence, for simplicity we assume that $a=1$, and (B) accordingly becomes (C):

(C) there exist positive numbers $p, q$ and $b$ such that $0<q<1<p<2^{*}-1, N(1-$ $q) /(1+q)=p-1$, and

$$
\limsup _{|t| \rightarrow 0}\left|\frac{g(x, t)-|t|^{q-1} t}{|t|^{p}}\right|<b<\min \left\{b_{0}, b_{1}\right\}
$$

uniformly in $x \in \Omega$.

Take a cut off function $\varphi(t)$ :

$$
\begin{cases}\varphi(t)=1, & \text { for all }|t| \leq t_{0} \\ 0<\varphi(t)<1, & t_{0}<|t|<2 t_{0} \\ \varphi(t)=0, & \text { otherwise. }\end{cases}
$$

Let $\tilde{g}(x, t)=\varphi(t) g(x, t)+(1-\varphi(t))|t|^{q-1} t$ for $(x, t) \in \Omega \times \mathbb{R}$. Then, $\widetilde{g}(x, t) \in \mathbf{C}(\Omega \times$ $\mathbb{R})$ and satisfying the following condition $(\mathbf{D})$ :

(D) there exist $t_{0}>0$ small and $b>0$ such that $b<\min \left\{b_{0}, b_{1}\right\}$,

$$
|t|^{q-1} t-b|t|^{p} \leq \widetilde{g}(x, t) \leq|t|^{q-1} t+b|t|^{p} \quad \text { for all }(x, t) \in \Omega \times \mathbb{R},
$$

and that $\tilde{g}(x, t)=|t|^{q-1} t$ for all $x \in \Omega$ with $t \geq 2 t_{0}$.

Throughout the rest paper, we consider the problem: $-\Delta u=\widetilde{g}(x, u), u \in H$ under (D). Equivalently, we first consider the problem (1.1) under the condition (E) :

(E) there exist $t_{0}>0$ small and $b>0$ such that $b<\min \left\{b_{0}, b_{1}\right\}$,

$$
|t|^{q-1} t-b|t|^{p} \leq g(x, t) \leq|t|^{q-1} t+b|t|^{p} \quad \text { for all }(x, t) \in \Omega \times \mathbb{R},
$$

and that $g(x, t)=|t|^{q-1} t$ for all $x \in \Omega$ with $t \geq 2 t_{0}$.

Here, we use many marks as the paper [8]. For each $k \geq 1$, we denote by $D^{k}$ and $S^{k-1}$ the unit disk and the unit sphere of $k$ dimensional Euclidian space, respectively. And we denote by $\langle\cdot, \cdot\rangle$ the inner product in $H$. For subsets $A, B$ of $H$ with $B \subset A$, we denote by $\pi_{k}(A, B)$ the k-relative homotopy group (cf. [10]). We denote by $B(r)$ the open ball of $H$ centred at 0 with radius $r$. For each functional $F: H \rightarrow R$ and $a \in R$, $F_{a}$ stands for the level set defined by $F_{a}=\{v \in H: F(v) \leq a\}$. We defined a functional 
$I: H \rightarrow R$ by

$$
I(u)=\frac{1}{2} \int_{\Omega}|\nabla u|^{2} d x-\frac{1}{q+1} \int_{\Omega}|u|^{q+1} d x, \quad \text { for all } u \in H .
$$

By $[\mathbf{8}]$, we know there exists some $N_{0}(v)>0$ satisfies

$$
I\left(N_{0}(v) v\right)=\min \{I(t v): t \geq 0\}<0,
$$

and one can see that for each $v \in H \backslash\{0\}$,

$$
\left\|N_{0}(v) v\right\|^{2}=\int_{\Omega}\left|N_{0}(v) v\right|^{q+1} d x \quad \text { and } \quad I\left(N_{0}(v) v\right)=\frac{q-1}{2(q+1)}\left\|N_{0}(v) v\right\|^{2} .
$$

We define functionals $J: H \rightarrow R$ and $\widehat{J}: H \rightarrow R$ by

$$
J(u)=\frac{1}{2} \int_{\Omega}|\nabla u|^{2} d x-\int_{\Omega} G(x, u(x)) d x \quad \text { for } u \in H
$$

and

$$
\widehat{J}(u)=\frac{1}{2} \int_{\Omega}|\nabla u|^{2} d x-\int_{\Omega} \frac{1}{q+1}|u|^{q+1}+\frac{b}{p+1}|u|^{p+1} d x \quad \text { for } u \in H .
$$

LEMMA 2.1. Under condition $(\mathbf{E})$, the functional $J(u)$ is coercive and the (PS) condition holds.

Proof. It is easy to show that there exists $C>0$ such that $G(x, v) \leq C+\frac{1}{q+1}|v|^{q+1}$, then $+\infty>J\left(u_{n}\right) \geq\left.\frac{1}{2}|| u_{n}\right|^{2}-C|\Omega|-\frac{1}{q+1}\left|u_{n}\right|_{q+1}^{q+1}$. Therefore, $\left\{u_{n}\right\}$ is bounded in $H$. Next, we may assume $u_{n} \rightarrow u$ in $H, u_{n} \rightarrow u$ in $L^{p+1}(\Omega)$. By $(\mathbf{E})$, we have $g\left(x, u_{n}\right) \rightarrow$ $g(x, u)$ in $L^{\frac{p+1}{p}}$ (see theorem A.2 of [12]).

Since $\left\langle J^{\prime}\left(u_{n}\right)-J^{\prime}(u), u_{n}-u\right\rangle \rightarrow 0$ and by Hölder inequality, we have

$$
\begin{aligned}
\left|\int_{\Omega}\left(g\left(x, u_{n}\right)-g(x, u)\right)\left(u_{n}-u\right) d x\right| & \leq\left|g\left(x, u_{n}\right)-g(x, u)\right|_{\frac{p+1}{p}}\left|u_{n}-u\right|_{p+1} \\
& \rightarrow 0
\end{aligned}
$$

Therefore,

$$
\left\|u_{n}-u\right\|^{2}=\left\langle J^{\prime}\left(u_{n}\right)-J^{\prime}(u), u_{n}-u\right\rangle+\int_{\Omega}\left(g\left(x, u_{n}\right)-g(x, u)\right)\left(u_{n}-u\right) d x=o(1) .
$$

The proof is completed.

LEMMA 2.2. For each $v \in H \backslash\{0\}$, there exists a unique positive number $N(v)<+\infty$ such that $\widehat{J}(N(v) v)$ is a local minimum value of $\{\widehat{J}(t v): t \geq 0\}$ with related to $t$ and $\widehat{J}(t v)$ decreases on $(0, N(v)]$.

REMARK 2.1. Since here $\widehat{J}$ has different form from [8], $b<b_{1}$ is needed to make sure that $N(v)<+\infty$, otherwise, $N(v)$ may not be well defined. Moreover, here we will indicate that the similar Lemma still holds. 
Proof. Let $v \in H \backslash\{0\}$ such that $\|v\|=1$. We put $c=\int_{\Omega}|v|^{q+1} d x$. Then,

$$
\begin{aligned}
f(t) & :=\frac{d}{d t} \widehat{J}(t v) \\
& =t\|v\|^{2}-t^{q} \int_{\Omega}|v|^{q+1} d x-b t^{p} \int_{\Omega}|v|^{p+1} d x \\
& =t|| v \|^{2}-c t^{q}-b t^{p}|v|_{p+1}^{p+1} \\
& =t\left[\left(\|v\|^{2}-b t^{p-1}|v|_{p+1}^{p+1}\right)-\frac{c}{t^{1-q}}\right] .
\end{aligned}
$$

For any $c_{1}, c_{2}>0$, let $h(t):=c_{1} t^{p-1}+c_{2} t^{q-1}$, then $h^{\prime}(t)=(p-1) c_{1} t^{p-2}-(1-q) c_{2} t^{q-2}$, so there exists unique $t_{v}=\left(\frac{c_{2}(1-q)}{c_{1}(p-1)}\right)^{\frac{1}{p-q}}>0$ such that $h^{\prime}\left(t_{v}\right)=0$, and one can see that $h^{\prime \prime}\left(t_{v}\right)>0$. This implies that

$$
\min \{h(t): t>0\}=h\left(t_{v}\right)=\left(\left(\frac{1-q}{p-1}\right)^{\frac{p-1}{p-q}}+\left(\frac{1-q}{p-1}\right)^{\frac{q-1}{p-q}}\right) c_{1}^{\frac{1-q}{p-q}} c_{2}^{\frac{p-1}{p-q}}
$$

Since $\Omega$ is bounded in $\mathbb{R}^{N}$ and $q+1, p+1<2^{*},\|v\|=1$, then $|v|_{p+1}^{p+1}<M^{p+1}$ and $|v|_{q+1}^{q+1}<M^{q+1}|\Omega|^{\frac{p-q}{p+1}}$. We let $c_{1}=b|v|_{p+1}^{p+1}$ and $c_{2}=|v|_{q+1}^{q+1}$. Recalling that $b<b_{1}$, we have

$$
\left(\left(\frac{1-q}{p-1}\right)^{\frac{p-1}{p-q}}+\left(\frac{1-q}{p-1}\right)^{\frac{q-1}{p-q}}\right) c_{1}^{\frac{1-q}{p-q}} c_{2}^{\frac{p-1}{p-q}}<1 .
$$

On the other hand, $h(t) \rightarrow+\infty$ as $t \rightarrow 0$ or $t \rightarrow+\infty$, so, there exist two numbers $0<$ $t_{0}<t_{v}<t_{1}$ such that $h\left(t_{0}\right)=h\left(t_{1}\right)=1$ and $h^{\prime}\left(t_{0}\right)<0, h^{\prime}\left(t_{1}\right)>0$. Therefore, $f^{\prime}\left(t_{0}\right)=$ $\left.\frac{d^{2}}{d t^{2}} \widehat{J}(t v)\right|_{t=t_{0}}>0$ and $f^{\prime}\left(t_{1}\right)=\left.\frac{d^{2}}{d t^{2}} \widehat{J}(t v)\right|_{t=t_{1}}<0$. This implies the uniqueness of $N(v)=t_{0}$ and the proof is completed.

REMARK 2.2. (improvement of Remark 1 in [8]) One can see that $N_{0}$ and $N$ are continuous. Since functions $I$ and $\widehat{J}$ are even, we have that $N_{0}$ and $N$ are even functions. Moreover, by the definition of $N_{0}(v)$ and $N(v), N(t v)=\frac{1}{t} N(v)$ and $N_{0}(t v)=\frac{1}{t} N_{0}(v)$ for all $t>0$.

Next, as in [8], we put $\beta_{k}=\min _{h \in \Gamma_{k} x \in S^{k-1}} I(h(x))$, where $\Gamma_{k}=\left\{h \in C\left(S^{k-1}, H\right): h(x)=\right.$ $-h(-x)$ for $\left.x \in \mathrm{S}^{\mathrm{k}-1}\right\}$. Then, we have the improvement of Lemma 2.2 in $[8]$ as the following:

LEMMA 2.3. (cf. [8]) Each $\beta_{k}$ is negative and there exist $k_{0} \geq 1$ such that

$$
\beta_{k} \geq-\left(\frac{1-q}{2(1+q)} T^{-\frac{1+q}{1-q}}|\Omega|\right) k^{\frac{2(q+1)}{N(q-1)}} \quad \text { for } k \geq k_{0} .
$$

Especially, for any $k \geq 1$ we have $\beta_{k+1} \geq \beta_{k}$.

Proof. The conclusion that each $\beta_{k}$ is negative and there exist some $C_{0}>0, k_{0} \geq 1$ such that $\beta_{k} \geq-C_{0} k^{\frac{2(q+1)}{N(q-1)}}$ for $k \geq k_{0}$ has been proved by Lemma 2.2 of [8]. Calculate carefully step by step, we can find an appropriate value that $C_{0}=\frac{1-q}{2(1+q)} T^{-\frac{1+q}{1-q}}|\Omega|$. It is trivial that $\beta_{k} \leq \beta_{k+1}$. 
We put $\alpha=\frac{p-1}{2}$ and $\gamma=\frac{2(q+1)}{N(1-q)}$, then $\gamma \alpha=1$. By Lemma 2.3 we can write $\beta_{k}=-m_{k} k^{-\gamma}$. For $k$ large enough, $0<m_{k} \leq C_{0}, m_{k+1} \leq m_{k}\left(\frac{k+1}{k}\right)^{\gamma}$. We also put $c:=\left\|N_{0}(v) v\right\|^{2}=\left|N_{0}(v) v\right|_{q+1}^{q+1}=\frac{2(q+1)}{q-1} I\left(N_{0}(v) v\right)$.

LEMMA 2.4. (cf. [8]) For each $v \in H \backslash\{0\}$ with $\left|I\left(N_{0}(v) v\right)\right|$ sufficiently small,

$$
J\left(N_{0}(v) v\right) \leq\left(1-\left(\left(\frac{2(q+1)}{1-q}\right)^{\frac{p+1}{2}} \frac{1}{p+1} b M^{p+1}\right)\left|I\left(N_{0}(v) v\right)\right|^{\frac{p-1}{2}}\right) I\left(N_{0}(v) v\right)
$$

Proof. Lemma 2.3 of [8] has showed that

$$
J\left(N_{0}(v) v\right) \leq\left(1-C_{1}\left|I\left(N_{0}(v) v\right)\right|^{\frac{p-1}{2}}\right) I\left(N_{0}(v) v\right) .
$$

Calculate carefully, we can find an appropriate value

$$
C_{1}=\left(\frac{2(q+1)}{1-q}\right)^{\frac{p+1}{2}} \frac{1}{p+1} b M^{p+1}
$$

REMARK 2.3. Lemma 2.4 is the inequality of (2.8) in [8], here we find a suitable value of $C_{1}$. Since there exist some mistakes in the proof of (2.9) in [8], next, we will give another way to prove more than (2.9) of [8] by the lemma 2.5.

LEMMA 2.5. For each $v \in H \backslash\{0\}$ with $\left|I\left(N_{0}(v) v\right)\right|$ sufficiently small,

$$
\begin{aligned}
\left(1+C_{2}\left|I\left(N_{0}(v) v\right)\right|^{\frac{p-1}{2}}\right) I\left(N_{0}(v) v\right) \leq \widehat{J}(N(v) v) & \leq I\left(N_{0}(v) v\right) \\
\leq & \left(1-C_{2}|\widehat{J}(N(v) v)|^{\frac{p-1}{2}}\right) \widehat{J}(N(v) v) .
\end{aligned}
$$

where

$$
C_{2}:=\left(\frac{2(q+1)}{1-q}\right)^{\frac{p-1}{2}} \frac{4 b(p+q+3)}{(1-q)(1+p)} M^{p+1}
$$

REMARK 2.4. (2.8),(2.9) of [8] is the source of our inspiration. However, essentially, (2.2) is different from (2.8), (2.9) of [8]. We obtain that the control of $I\left(N_{0}(v) v\right)$ and $\widehat{J}(N(v) v)$ is mutual. Note (2.11) of [8], we could delete $(2.11)$ and take $\lambda^{2}\left(\frac{q-1}{2(q+1)}\|v\|^{2}-\right.$ $\left.\frac{2 b}{p+1} M^{p+1}\|v\|^{p+1}\right)$ in place of (2.12) in [8]. Furthermore, our goal is to point out that $C_{2}$ in (2.2) can be taken some sensible values under (E).

Proof. Let $v \in H \backslash\{0\}$ such that $N(v)=1$ and suppose that $\left|I\left(N_{0}(v) v\right)\right|$ is sufficiently small, since $I\left(N_{0}(v) v\right)=\frac{q-1}{2(q+1)}\left\|N_{0}(v) v\right\|^{2}$, then, $c$ is sufficiently small.

Let

$$
f(t):=\frac{d}{d t} \widehat{J}(t v)=t|| v \|^{2}-t^{q} \int_{\Omega}|v|^{q+1} d x-b t^{p} \int_{\Omega}|v|^{p+1} d x .
$$


Since $N(v)=1$, we have $f(1)=0$, that is, $\left.|| v\right|^{2}-|v|_{q+1}^{q+1}-b|v|_{p+1}^{p+1}=0$. Then,

$$
\begin{aligned}
N_{0}(v)^{2}\|v\|^{2} & =N_{0}(v)^{q+1}|v|_{q+1}^{q+1} \\
& =N_{0}(v)^{q+1}\left(\|v\|^{2}-b|v|_{p+1}^{p+1}\right) \\
& <N_{0}(v)^{q+1}|| v \|^{2}
\end{aligned}
$$

which implies

$$
N_{0}(v)<1
$$

On the other hand, since $\left\|N_{0}(v) v\right\|^{2}=\left|N_{0}(v) v\right|_{q+1}^{q+1}$, we have

$$
\begin{aligned}
\|v\|^{2}-N_{0}(v)^{1-q}\|v\|^{2} & =\|v\|^{2}-|v|_{q+1}^{q+1} \\
& =b|v|_{p+1}^{p+1} \\
& \leq b M^{p+1}\|v\|^{p+1}
\end{aligned}
$$

which implies

$$
N_{0}(v)^{p-1}-N_{0}(v)^{p-q} \leq b M^{p+1} c^{\frac{p-1}{2}} .
$$

Then, we have one of the following holds :

(1) $0<N_{0}(v)$ is sufficiently small;

(2) $0<1-N_{0}(v)$ is sufficiently small.

But by Lemma 2.2 we have $N(v)=t_{0}<t_{v}$, that is,

$$
1=N(v)<\left(\frac{(1-q)|v|_{q+1}^{q+1}}{(p-1) b|v|_{p+1}^{p+1}}\right)^{\frac{1}{p-q}}
$$

Then,

$$
\begin{aligned}
(1-q)|v|_{q+1}^{q+1} & >(p-1) b|v|_{p+1}^{p+1} \\
& =(p-1)\left(|| v \|^{2}-|v|_{q+1}^{q+1}\right),
\end{aligned}
$$

which implies $N_{0}(v)>\left(\frac{p-1}{p-q}\right)^{\frac{1}{1-q}}$. Therefore, case (1) is impossible, then $1-N_{0}(v)$ is sufficiently small, and we have

$$
\begin{aligned}
\frac{1}{2}\left(1-N_{0}(v)^{1-q}\right) & <\left(1-N_{0}(v)^{1-q}\right) N_{0}(v)^{p-1} \\
& =N_{0}(v)^{p-1}-N_{0}(v)^{p-q} \\
& \leq b M^{p+1} c^{\frac{p-1}{2}}
\end{aligned}
$$

Thus, we get

$$
\left(1-2 b M^{p+1} c^{\frac{p-1}{2}}\right)^{\frac{1}{1-q}}<N_{0}(v)<1 .
$$


Note that $\widehat{J}(t v) \leq I(t v)$ always holds. Then, by (2.5) and lemma 2.2,

$$
\widehat{J}(v) \leq \widehat{J}\left(N_{0}(v) v\right) \leq I\left(N_{0}(v) v\right)<0 .
$$

That is,

$$
\widehat{J}(N(v) v) \leq \widehat{J}\left(N_{0}(v) v\right) \leq I\left(N_{0}(v) v\right)<0 .
$$

Next, we take $v \in H \backslash\{0\}$ such that $N_{0}(v)=1$, and put $\lambda=N(v)$. By Remark 2.2, we have that $N_{0}(v) v=N_{0}(\lambda v) \lambda v$ and $N(\lambda v)=1$. Thus $c=\left\|N_{0}(v) v\right\|^{2}=\left\|N_{0}(\lambda v) \lambda v\right\|^{2}$, then by (2.5),

$$
\left(1-2 b M^{p+1} c^{\frac{p-1}{2}}\right)^{\frac{1}{1-q}}<N_{0}(\lambda v)=\frac{N_{0}(v)}{\lambda}=\frac{1}{\lambda}<1 .
$$

When $c$ is sufficiently small, we have that

$$
1<\lambda<\left(1+b^{\prime} M^{p+1} c^{\frac{p-1}{2}}\right)^{\frac{1}{1-q}},
$$

where $b^{\prime}:=\frac{3}{2} b>b$. Then, we have $1<\lambda^{p-1}<2$ and by the Taylor formula that

$$
\lambda^{2} \leq 1+\frac{4 b}{1-q} M^{p+1} c^{\frac{p-1}{2}} .
$$

Hence,

$$
\begin{aligned}
\widehat{J}(\lambda v) & =\frac{1}{2}\|\lambda v\|^{2}-\frac{\lambda^{q+1}}{q+1} \int_{\Omega}|v|^{q+1}-\frac{\lambda^{p+1} b}{p+1} \int_{\Omega}|v|^{p+1} \\
& =\lambda^{2}\left(\frac{1}{2} c-\frac{\lambda^{q-1}}{q+1} c-\frac{\lambda^{p-1} b}{p+1} \int_{\Omega}|v|^{p+1}\right) \\
& \geq \lambda^{2}\left(\frac{1}{2} c-\frac{1}{q+1} c-\frac{2 b}{p+1} \int_{\Omega}|v|^{p+1}\right) \\
& =\lambda^{2}\left(\frac{q-1}{2(q+1)}\|v\|^{2}-\frac{2 b}{p+1} M^{p+1}\|v\|^{p+1}\right)
\end{aligned}
$$

Then,

$$
\widehat{J}(\lambda v) \geq \lambda^{2}\left(1+c_{1}\|v\|^{p-1}\right) I(v)
$$

where $c_{1}=\frac{4 b(q+1)}{(1-q)(1+p)} M^{p+1}$. By (2.7), (2.8) and Taylor formula, we have $(1+$ $\left.c_{2}|I(v)|^{\frac{p-1}{2}}\right) I(v) \leq \widehat{J}(\lambda v)<0$, here we let

$$
c_{2}:=\left(\frac{2(q+1)}{1-q}\right)^{\frac{p-1}{2}} \frac{4 b(p+q+3)}{(1-q)(1+p)} M^{p+1} .
$$

We put $C_{2}=c_{2}$, then,

$$
\left(1+C_{2}\left|I\left(N_{0}(v) v\right)\right|^{\frac{p-1}{2}}\right) I\left(N_{0}(v) v\right) \leq \widehat{J}(N(v) v) \leq I\left(N_{0}(v) v\right) .
$$


Let $x=\left|I\left(N_{0}(v) v\right)\right|, y=|\widehat{J}(N(v) v)|$, we have $0<x \leq y \leq\left(1+C_{2} x^{\alpha}\right) x$. Then, $\left|\frac{y-x}{y^{\alpha+1}}\right| \leq$ $C_{2}$. Hence, $\widehat{J}(N(v) v) \leq I\left(N_{0}(v) v\right) \leq\left(1-C_{2}|\widehat{J}(N(v) v)|^{\frac{p-1}{2}}\right) \widehat{J}(N(v) v)$.

REMARK 2.5. By (2.3), we know that if $N(v)=1$, then $N_{0}(v)<1$. By (2.6), if $N_{0}(v)=1$, then $N(v)>1$.

Let

$$
C_{3}:=2\left(C_{1}+C_{2}\right)=\left(\frac{2(q+1)}{1-q}\right)^{\frac{p-1}{2}} \frac{8 p+12 q+28}{(1-q)(1+p)} b M^{p+1}>0,
$$

then similarly to [8], we can choose $m>0$, such that for each $t \in(-m, 0)$,

$$
\left(1-C_{2}\left[\left(1-C_{1}|t|^{\alpha}\right)|t|\right]^{\alpha}\right)\left(1-C_{1}|t|^{\alpha}\right) t<\left(1-C_{3}|t|^{\alpha}\right) t .
$$

Indeed, if we let

$$
h(t):=\frac{1-\left(1-C_{2}\left[\left(1-C_{1}|t|^{\alpha}\right)|t|\right]^{\alpha}\right)\left(1-C_{1}|t|^{\alpha}\right)}{|t|^{\alpha}},
$$

then we have $\lim _{t \rightarrow 0} h(t)=C_{1}+C_{2}>0$. Hence, we can find $m>0$ such that for each $t \in(-m, 0), 0<h(t)<C_{3}$, then we have

$$
\left(1-C_{2}\left[\left(1-C_{1}|t|^{\alpha}\right)|t|\right]^{\alpha}\right)\left(1-C_{1}|t|^{\alpha}\right) t<\left(1-C_{3}|t|^{\alpha}\right) t .
$$

REMARK 2.6. Assume that (E) holds, we have that $C_{3} C_{0}^{\alpha}<\frac{\gamma}{2}$ and $C_{3} C_{0}^{\alpha} \alpha<\frac{1}{2}$.

LEMmA 2.6. $\forall 0<\theta$ there exists a sequence $\left\{k_{i}\right\} \subset N$ such that $k_{i} \rightarrow \infty$ as $i \rightarrow \infty$, and $m_{k_{i}+1}<m_{k_{i}}\left(\frac{k_{i}+1}{k_{i}}\right)^{\theta}$

Proof. Suppose that, if there exists some $k_{0} \geq 1$ such that, $m_{k+1} \geq m_{k}\left(\frac{k+1}{k}\right)^{\theta}$ for all $k \geq k_{0}$. Then, $\frac{m_{n k_{0}}}{m_{k_{0}}} \geq n^{\theta}$, which implies when $n$ is large enough $m_{n k_{0}}>m_{k_{0}} n^{\theta} \rightarrow+\infty$. This is a contradiction to $m_{k} \leq C_{0}$.

Lemma 2.7. There exists a sequence $\left\{k_{i}\right\} \subset N$ such that $k_{i} \rightarrow \infty$ as $i \rightarrow \infty$, and

$$
\beta_{k_{i}+1}>\left(1-C_{3}\left|\beta_{k_{i}}\right|^{\alpha}\right) \beta_{k_{i}} \text { for all } i \geq 1
$$

REMARK 2.7. When $\frac{1}{\alpha}<\gamma$, the same conclusion has been showed in [8] by an useful inequality of [3] which depends on the strict inequality $\frac{1}{\alpha}<\gamma$ holds. In [8], the author used the truncation approach. Thanks to the strict inequality $\frac{1}{\alpha}<\gamma$, it play an crucial role in the process of dealing with inequalities that the exponent can be share a very little with the cut off level $t_{0}$ small. At the present paper, we still use the truncation approach, and consider that $g(x, t)=|t|^{q-1} t$ for all $x \in \Omega$ with $t \geq 2 t_{0}$. But the real essential condition for problem (1.1) is that $b$ is small enough since we cannot share any part from the exponent. Hence there are many inequalities seem like those in [8], but there are a lot of differences in the process of dealing with these inequalities. 
Proof. Let $\theta=\frac{\gamma}{2}>0$, by lemma 2.6,

$$
\begin{aligned}
\frac{-m_{k_{i}+1}+m_{k_{i}}\left(\frac{k_{i}+1}{k_{i}}\right)^{\gamma}}{m_{k_{i}}^{\alpha+1} k_{i}^{-\gamma \alpha}} \frac{k_{i}^{\gamma}}{\left(k_{i}+1\right)^{\gamma}} & =\frac{-\frac{m_{k_{i}+1}}{m_{k_{i}}}+\left(\frac{k_{i}+1}{k_{i}}\right)^{\gamma}}{m_{k_{i}}^{\alpha} k_{i}^{-\gamma \alpha}} \frac{k_{i}^{\gamma}}{\left(k_{i}+1\right)^{\gamma}} \\
& >\frac{\left(\frac{k_{i}+1}{k_{i}}\right)^{\gamma}-\left(\frac{k_{i}+1}{k_{i}}\right)^{\frac{\gamma}{2}}}{k_{i}^{-\gamma \alpha}} \frac{1}{m_{k_{i}}^{\alpha}}\left(\frac{k_{i}}{k_{i}+1}\right)^{\gamma} \\
& >\frac{\gamma}{2 C_{0}^{\alpha}} \text { when } k_{i} \text { is large enough. }
\end{aligned}
$$

By Remark 2.6, $\frac{\gamma}{2 C_{0}^{\alpha}}>C_{3}$, which implies that when $k_{i}$ is large enough,

$$
\beta_{k_{i}+1}>\left(1-C_{3}\left|\beta_{k_{i}}\right|^{\alpha}\right) \beta_{k_{i}}
$$

LEMMA 2.8. (cf. [8]) For each $i \geq 1$, there exist $\varepsilon_{i}>0$ and $c<0$ such that $\beta_{k_{i}}+\varepsilon_{i}<$ $c$ and $\pi_{k_{i}}\left(J_{c}, J_{\beta_{k i}+\varepsilon}\right) \neq\{0\}$ for $0<\varepsilon<\varepsilon_{i}$.

Proof. The details are similar to that of Lemma 2.6 in [8].

REMARK 2.8. Since $N(v) v$ is a local minimizer of $\widehat{J}(t v)$ in this paper but it is a global minimizer in [8], $\widehat{J}(t v)$ decreases on $(0, N(v)]$ and Remark 2.5 are necessary. If not, the similar inequality of (2.15) in [8] may not hold. In fact replaces $\widehat{J}(N(v) v)$ by $J(N(v) v)$, one can show that (2.2) still holds, but we can not ensure that $J(t v)$ decreases on $(0, N(v)]$. In another word, we can not ensure $J(N(f(z)) f(z)) \leq d_{k}$. Therefore, we have to introduce the functional $\widehat{J}$.

Proof of Theorem 1.1. Next, we can get sequences $\left\{k_{i}\right\},\left\{c_{i}\right\},\left\{e_{i}\right\}$ and $\left\{\varepsilon_{i}\right\}$ such that $\beta_{k_{i}}+\varepsilon_{i}<e_{i}<0$ are regular values of $J$, where $c_{i}=\min _{h \in[\sigma]_{x \in S_{+}^{k_{i}}}} J(h(x))$ with $[\sigma] \in$ $\pi_{k_{i}}\left(J_{e_{i}}, J_{\beta_{k_{i}}+\varepsilon_{i}}\right)$ nontrivial, $c_{i}>\beta_{k_{i}}+\varepsilon_{i}$ and $J$ satisfies the $(P S)_{c_{i}}$ condition. Therefore, we by Theorem 1.4 of [5] , there exists a sequence of critical points $\left\{u_{i}\right\} \subset H$ of $J$ with critical value $\left\{c_{i}\right\}$ and $c_{i} \in\left(\beta_{k_{i}}+\varepsilon_{i}, 0\right)$. Then, we have $c_{i} \rightarrow 0$. Hence, under condition $(\mathbf{E})$, the conclusions of theorem 1.1 hold. Equivalently, we have discussed the problem: $-\Delta u=\tilde{g}(x, u)$ under (D). The remaining work we need to do is the bootstrap argument. At last, we can find that $\lim _{i \rightarrow \infty}\left|u_{i}\right|_{\infty}=0$. Recalling that $\tilde{g}(x, t)=g(x, t)$ for all $x \in \Omega$ with $|t| \leq t_{0}$, we obtain that $u_{i}$ is a solution of Problem (1.1) for $i$ is large enough (the details see $[\mathbf{8}]$ ). Hence, theorem 1.1 holds.

\section{REFERENCES}

1. A. Ambrosetti, H. Brezis and G. Cerami, Combined effects of concave and convex nonlinearities in some elliptic problems, J. Funct. Anal. 122 (1994), 519-543.

2. A. Ambrosetti and M. Badiale, The dual variational principle and elliptic problems with discontinuous nonlinearities, J. Math. Anal. Appl. 140 (1989), 363-373.

3. A. Bahri and H. Berestycki, A perturbation method in critical point theory and applications, Trans. Amer. Math. Soc. 267 (1981) 1-31.

4. T. Bartsch and M. Willem, On an elliptic equations with convex and concave nonlinearties, Proc. Amer. Math. Soc. 123 (1995), 3555-3561. 
5. K. C. Chang, Infinite dimensional morse theory and multiple solution problems (Birkhäuser, 1993).

6. R. Courant and D. Hilbert, Methods of mathematical physics, Vol. I (Inerscience, New York, 1953).

7. J. Garcia Azorero and I. Peral Alonso, Multiplicity of solutions for elliptic problems with critical exponent or with a nonsymmetric term, Trans. Amer. Math. Soc. 323 (1991), 877-895.

8. N. Hirano, Existence of infinitely many solutions for sublinear elliptic problems, $J$. Math. Anal. Appl. 218(2003), 83-92.

9. R. Kajikiya, Non-radial solutions with group invariance for the sublinear EmdenFowler equation, Nonlinear Anal. 28 (2002), 567-597.

10. E. Spanier, Algebraic topology (McGraw-Hill, New York, 1966).

11. Z. Q. Wang, Nonlinear boundary value problems with concave nonlinearities near the origin, Nonlinear differ. equ. appl., 8 (2001) 15-33.

12. M. Willem, Minimax theorems (Birkhäuser, 1996). 